\title{
Systemic Hemodynamic Atherothrombotic Syndrome: Expanding the Concept
}

Evasandrid Romero-Jimenez ${ }^{1, \dagger}$, Jesús David Moreno-Gamarra ${ }^{2, \dagger}$, Kenia Alexandra Villalba-Ortega ${ }^{1, \dagger}$ and Md Moshiur $\operatorname{Rahman}^{3, * \dagger}$

${ }^{1}$ School of Medicine, University of Cartagena, Cartagena, Colombia

${ }^{2}$ School of Medicine, Universidad del Sinú, Cartagena, Colombia

${ }^{3}$ Department of Neurosurgery, Holy Family Red Crescent Medical College, Dhaka, Bangladesh

${ }^{\dagger}$ Equally contributed authors

*Corresponding author: Md Moshiur Rahman, Department of Neurosurgery, Holy Family Red Crescent Medical College, Dhaka, Bangladesh

Received date: 1 November, 2021 I Accepted date: 8 November, 2021 | Published date: 11 November, 2021

Citation: Jimenez ER, Gamarra JDM, Ortega KAV, Rahman MM. (2021) Systemic Hemodynamic Atherothrombotic Syndrome: Expanding the Concept. J Anaesth Anesth Drug 1(1). doi https://doi.org/10.54289/JAAD2100103

Copyright: () 2021 Jimenez ER, et al. This is an open-access article distributed under the terms of the Creative Commons Attribution License, which permits unrestricted use, distribution, and reproduction in any medium, provided the original author and source are credited.

Abbreviations: MBPS: Morning Blood Pressure Surge, LVH: left ventricular hypertrophy, SHATS: systemic hemodynamic atherothrombotic syndrome, BP: blood pressure.

\section{Dear editor,}

With the advance of translational medicine, it has been possible to describe new pathophysiological mechanisms of the diseases that generate the greatest burden of disease globally, one of these, the cardiovascular diseases. Recently, was published an article where the authors evaluated the impact of Morning Blood Pressure Surge (MBPS) on the development of left ventricular hypertrophy (LVH) as a marker of cardiovascular morbidity in normotensive patients with type II diabetes mellitus [1]. The authors observed a positive and statistically significant correlation $(\mathrm{p}<0.001)$ between MBPS and LVH. Ibrahim et al [1] conclude that MBPS is an independent factor of LVH, and that it is also associated with diastolic dysfunction in normotensive patients with type II diabetes mellitus. We thank the authors for providing such valuable evidence. However, we would like to present the concept of systemic hemodynamic atherothrombotic syndrome (SHATS), a recently described pathological condition that integrates the observations presented by the authors, and which also allows us to estimate the real risk of cardiovascular morbidity in patients with underlying cardiometabolic disease.

Systemic hemodynamic atherothrombotic syndrome, a relatively new entity described by Dr. Kazuomi Kario [2], consists of a vicious synergistic cycle of hemodynamic stress and vascular disease [2]. Currently, it has a score for the diagnosis and evaluation of its severity [2]. The interesting thing about this syndrome is that it considers blood pressure (BP) dysregulation as the main axis, represented by the variability generated by certain factors such as climate, season, environment, home, work, emotional state, alcohol, among others; on BP [2,3]. It is based on early vascular aging and acute arterial damage, due to altered blood flow and sudden BP variability, causing silent target organ damage (e.g., heart) $[2,3]$.

His approach initially arose from the hypothesis of resonance of blood pressure variability [3], when he observed that in detail, disorders in the neurobiology of BP, endocrine and epigenetic factors, exhibited phenotypes such as the shortest 
beat-by-beat variability, diurnal variability changes modified by position, diet or physical activity; day-by-day variability, visit-to-visit variability, seasonal variability (in which BP is higher winter and lower in summer), and the longest yearly variability of BP [3]. Based on the above, in an extraordinary way, Kario K [3] affirms that "the resonance of each type of BP variability with different time phases synergistically generates an extraordinarily large dynamic surge which would trigger a cardiovascular event [3]. From here arises the primary concept of "resonance hypothesis of BP variability" [3].

The creator of SHATS himself argues that morning surge in blood pressure is a phenotype of SHATS, so it is not an isolated event, but a marker of neuroendocrine dysregulation, endothelial dysfunction and therefore, vascular disease with risk of severe cardiovascular events [4]. Thus, morning surge in blood pressure itself is an independent marker of cardiovascular risk in normotensive patients, since even if the criteria for essential hypertension are not met, it represents established vascular disease and target organ damage [4].

Now, this correlates with the findings of Ibrahim et al [1], since the group of patients evaluated were diabetic, i.e., they had a history of cardiometabolic disease that substantially affects endothelial health and generates endocrine disruption [5]. Even if patients are not diagnosed with hypertension, vascular aging induced by type II diabetes mellitus, age and any other metabolic factor such as obesity or smoking, will cause the presence of SHATS, expressed through the phenotype: morning surge in blood pressure [4]. For this reason, it has been postulated to initiate treatment aimed at normalization of blood pressure variability and strict control of persistent hyperglycemic state is recommended [5]. Although the group evaluated by Ibrahim et al [1] did not have diagnosed essential hypertension, vascular injury, target organ damage (LVH) and the risk of triggering a major cardiovascular event existed by SHATS, therefore, this concept should be taken into account to modify risk score, accelerate diagnosis and treatment of people with silent vascular disease.
We suggest that future studies consider the plausible arguments for this syndrome, which encompasses more.

Factors such as time of day, place of work or home, seasons, psychological stress, environment, among many others; in order to find more evidence to answer questions that currently exist about the evolution of blood pressure, hemodynamic dynamics, endothelial dysfunction, insulin resistance and hyperglycemia, components that modify the evolution and prognosis of hypertension and type II diabetes mellitus, two of the chronic noncommunicable diseases with the highest burden of global disease, costs to health systems and impact on the quality of life of those affected [5].

\section{Conflict of Interest}

The authors declare that they have no conflicts of interest

\section{Reference}

1. Ibrahim IM, Abdel-Kareem ARO, Mahfouz RA, et al. (2021) The Association Between Morning Blood Pressure Surge and Cardiovascular Disease in Normotensive Type 2 Diabetic Patients: Observational Analytical Study in the Form of a Cross-Sectional Study. SN Compr Clin Med. 3: 955-963.

2. Kario K, Chirinos JA, Townsend RR, Weber MA, Scuteri A, et al. (2020) Systemic hemodynamic atherothrombotic syndrome (SHATS) - Coupling vascular disease and blood pressure variability: Proposed concept from pulse of Asia. Prog Cardiovasc Dis. 63(1): 22-32.

3. Kario K. (2016) Systemic Hemodynamic Atherothrombotic Syndrome and Resonance Hypothesis of Blood Pressure Variability: Triggering Cardiovascular Events. Korean Circ J. 46(4): 456-467.

4. Kario K. (2015) Morning surge in blood pressure: a phenotype of systemic hemodynamic atherothrombotic syndrome. Am J Hypertens. 28(1): 7-9.

5. Glovaci D, Fan W, Wong ND. (2019) Epidemiology of Diabetes Mellitus and Cardiovascular Disease. Curr Cardiol Rep. 21(4): 21. 